\title{
Study on the Safety and Efficacy of Indonesian Combination Plant Extract in Cosmetics
}

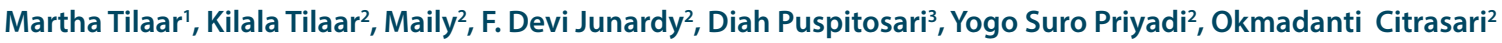 \\ 'Founder of Martha Tilaar Group, INDONESIA. \\ ${ }^{2}$ Martha Tilaar Innovation Centre, Jakarta, INDONESIA. \\ ${ }^{3}$ Dermatologist, Dermatology and Venereology Department, Medical Faculty, Universitas Padjajaran, Bandung, INDONESIA
}

\begin{abstract}
Objective: To look for antioxidant, lightening and anti aging effects from combination of Indonesian plant consist of Centella asiatica, Camellia sinensis and Glycine max extract in propanediol (CCGE). Methodology: The study was carried out using DPPH scavenging activity for antioxidant effect and anti tyrosinase activity for lightening effect. The efficacy test on volunteers was performed using Mexameter MX 16 and Cutometer MPA 580 to measure skin melanin index, elasticity and firmness respectively. The dermatological safety test was conducted using Repeated Opened Patch Test (ROPT) and Single Closed Patch Test (SCPT), while HETCAM test was performed to evaluate irritation at ocular level. Result: At the concentration of $2 \%$, CCGE has DPPH scavenging activity and anti tyrosinase activity of $70.56 \%$ and $52.76 \%$ respectively compared to ascorbic acid and kojic acid. The efficacy test showed that CCGE significantly increased skin elasticity and firmness after two and four weeks of application compared to base, but showed no significant effect on skin lightening. The dermatological safety test showed that CCGE did not cause any irritation or allergic skin reaction at the concentration of $5 \%$ for the ROPT and up to $3 \%$ for the
\end{abstract}

SCPT. Meanwhile at the concentration of 5\%, CCGE could cause allergic reaction in $4.0 \%$ of all subjects. The HETCAM test showed that CCGE is not irritant at the ocular level. Conclusion: These results concluded that CCGE is proven to be safe for human skin as well as at ocular level and could be used as cosmetic active ingredient for anti aging.

Key words: Centella asiatica, Camellia sinensis, Glycine max, Antioxidant, Lightening, Anti aging, Cosmetic.

\author{
Correspondence : \\ Maily \\ Martha Tilaar Innovation Centre, Jakarta, INDONESIA. \\ Phone no: 62-21-4603717 \\ Email: maily@marthana.co.id \\ DOI: $10.5530 /$ jyp.2017.1s.14
}

\section{INTRODUCTION}

Natural cosmetic ingredients have shown tremendous growth in recent years, ${ }^{1}$ as the demand for naturally derived active ingredient continues to increase globally along with the trend "back to nature". Compare to cosmetic products with synthetic chemicals, natural cosmetic products are safer to use, have relatively less side effect, and more compatible with all skin types. ${ }^{2}$

Indonesia is the biggest archipelago $^{3}$ in the world with an extraordinary biodiversity which been providing the populations with uncountable sources of ingredients that are used traditionally to treat ailments and health discomforts as well as for beauty care. They used various parts of local plants and trees, from roots, tubers, leaves, flowers, fruits, seeds, and even barks and twigs. This indicates that actually the concept of "live with nature" has long been embraced as Indonesian philosophy of life since ancient time.

The aim of this study is to examine the safety and efficacy of a combination of Indonesian plant extracts for cosmetic use. Several aspects should be considered when utilizing botanical materials in cosmetic, such as, the quality of the plant materials, process, biological activity, and safety consideration.

\section{MATERIAL AND METHODS}

\section{MATERIALS}

\section{Plants materials}

Gotu kola (Centella asiatica) dried herb, tea (Camellia sinensis) dried leaf and soybean (Glycine max) dried seed obtained from a community plantation on a Javanese island were used for this study. A taxonomical study was conducted by literature.

\section{Materials and reagents}

To prepare plant extract, the following materials were used: ethanol (Molindo Raya), demineralised water (Martina Berto, PT), propanediol (DuPont Tate \& Lyle Bioproduct), and phenoxetol (Clariant). 1,1-Diphenyl-2-picylhadrazyl (DPPH) (sigma) and methnol (Merck) were used in measuring the antioxidant activity with $\mathrm{L}(+)$ ascorbic acid (Merck) as positive control. L-tyrosine (Sigma), tyrosinase (Sigma), sodium dyhydrogen phospate monohydrate (Merck), and disodium hydrogen phospate (Merck) were used in measuring the anti tyrosinase activity with Kojic acid (Sigma) as positive control.

\section{Equipment}

Electronic balance (Mettler Toledo), rotary evaporator (Buchii), spectrofotometer UV-16001 (Shimadzu), waterbath (Memmert), loupe for observing the skin condition in the dermatological safety test. Mexameter 
MX 16 was used for measuring skin melanin index, while Cutometer MPA 580

\section{METHODS}

\section{In-Vitro Eye Irritation \& Dermatological Safety Testing Material}

Fresh fertile white leghorn eggs were used in In-vitro eye irritation testing and finn chamber with tape as tools to apply extract on human skin on dermatological safety testing.

Preparation of Extract

Gotu kola (Centella asiatica) dried herb, tea (Camellia sinensis) dried leaf and soybean (Glycine max) dried seed was extracted in ethanol. This extract was evaporated into dryness using rotary evaporator in vacuum. Following that, dried extract was diluted in propanediol to obtain the final extract (CCGE).

\section{Antioxidant Activity ${ }^{4}$}

The antioxidant activity was determined according to the method of Shimada et.al. using DPPH radical scavenging activity 3). $0.2 \mathrm{~mL}$ of CCGE in methanol and L $(+)$ ascorbic acid in demineralized water was added to freshly-prepared methanolic solution of DPPH. Each mixture was then shaken and incubated at room temperature for 30 minutes before it was spectrophotometrically measured at $517 \mathrm{~nm}$. The percentage of DPPH scavenging activity of each plant extract and L (+) ascorbic acid is expressed by:

$\%$ Scavenging activity $=1-\frac{\text { test sample absorbance }}{\text { blank sample absorbance }} \times 100$

\section{Anti Tyrosinase Activity ${ }^{5}$}

The whitening activity was determined in vitro according to the method of Vanni et.al. using L-tyrosine solution as the substrate 4). Two $\mathrm{mL}$ of CCGE and kojic acid solution in phosphate buffer was added with 0.5 $\mathrm{mL}$ L-tyrosine solution and $0.5 \mathrm{~mL}$ tyrosinase solution or $0.5 \mathrm{~mL}$ phosphate buffer. Each mixture was then shaken and incubated at $37 \mathrm{OC}$ for 60 minutes before it was spectrophotometrically detected at $475 \mathrm{~nm}$. The percentage of tyrosinase inhibition rate of CCGE and kojic acid is expressed by:

$$
\% \text { tyrosinase inhibition rate }=\frac{\mathrm{Ab}-(\mathrm{At}-\mathrm{AO})}{\mathrm{Ab}} \times 100
$$

where $\mathrm{Ab}$ is the absorbance of blank sample, At is the absorbance of sample with tyrosinase and Ao is the absorbance of sample without tyrosinase

\section{Dermatological Safety Test ${ }^{6-8}$}

Fifty (50) selected female subjects according to below criteria were recruited for this study.

Inclusion Criteria: The subjects who participated in this study had to be 18-54 years old with healthy and normal and/or sensitive skin. Prior to the study, they had to sign informed consent form that contains information regarding the study including the benefits and risks for them that may occur during the study. They also had to refrain from using any kind of products on their back skin except products that part of the study, one week before and during the study.

\section{Exclusion Criteria}

Subjects with skin and/or medical problem cannot be enrolled in the study. Those subjects with pregnancy, on lactation period, and/or using oral/topical application of medicine that can influence the skin condition were also excluded.

\section{Repeated Opened Patch Test (ROPT)}

CCGE with concentration of $5 \%$ was applied on to the skin area of the back. During application, the area was left unwashed. The skin reactions were then evaluated by trained assessor after 24 hours, 48 hours and 72 hours after application. The reactions observed were skin irritation and allergies, objectively as well as subjectively.

\section{Single Closed Patch test (SCPT)}

CCGE with concentration of $1 \%, 3 \%$ and $5 \%$ were applied each under occlusive patch on the suitable area of the skin on the back. During application, the area was left unwashed. The patches were removed after $24 \mathrm{~h}$ of application. The skin reactions were then evaluated by trained assessor after 1 hour, $24 \mathrm{~h}, 48 \mathrm{~h}$ and $72 \mathrm{~h}$ after patches removal.

\section{Evaluation Method}

The skin reactions were evaluated based on duration and the severity level of the reaction. The observed phenomena were scored according to scale developed by the International Contact Dermatitis Research Group

\section{HETCAM test ${ }^{9}$}

To evaluate the irritation level of CCGE to mucous membrane, HETCAM test was conducted according to the method of Luepke. 5\% of CCGE in demineralised water was applied onto the chorioallantoic membrane and left in contact for 20 seconds. The membrane was then evaluated for 5 minutes for any appearance of hyperemia, hemorrhage, and opacity.

\section{Efficacy Evaluation ${ }^{10}$}

The whitening, and elasticity and firmness effect of CCGE and base were evaluated clinically. Twenty- eight selected female subjects were involved. They were recruited according to below criteria.

Inclusion Criteria: The subjects who participated in this study has to be 18-35 years old with healthy and normal to dry skin. Prior to the study, they had to sign an informed consent form that contains information regarding the study including the benefits and risks for them that may occur during the study. They also had to refrain from using any kind of products on their face except products that part of the study, one week before and during the study.

Exclusion Criteria: Subjects with skin and/or medical problem cannot be enrolled in the study. Those subjects with pregnancy, on lactation period, and/or using oral/topical application of medicine that can influence the skin condition were also excluded.

\section{Evaluation on Skin Melanin Index}

Initial/baseline evaluation on skin melanin index was conducted by using Mexameter MX 16. Then, according to randomization scheme CCGE and base were applied on each facial skin twice a day for 4 weeks. Other evaluations were taken place at 2 weeks after application.

\section{Evaluation on Skin Elasticity \& Firmness}

Initial/baseline evaluation on skin elasticity \& firmness was conducted by using Cutometer MPA 580. Then, according to randomization scheme CCGE and base were applied on each facial skin twice a day for 4 weeks. Other evaluations were taken place at 2 weeks after application. 


\section{RESULTS AND DISCUSSION}

\section{Antioxidant Activity}

The result showed that CCGE at concentration of $2 \%$ had DPPH scavenging activity of $68.37 \%$ with $70.56 \%$ relative potency to ascorbic acid as the reference substance. This result indicate that CCGE has antioxidant activity against free radical of DPPH and therefore it has a potency to be antioxidant agent.

\section{Anti Tyrosinase Activity}

The test showed that CCGE at concentration of $2 \%$ had tyrosinase inhibitory activity with inhibition rate of $52.06 \%$ and potency ratio of $52.76 \%$ compared to kojic acid as the reference substance. This result indicate that CCGE has anti tyrosinase activity and therefore has potency to be whitening agent.

\section{Dermatological Safety Test}

In the dermatological safety test, fifty subjects aged between $18-54$ years old had completed the study, which consisted of 22 subjects with sensitive skin and 28 subjects with normal skin. The complete result can be seen on Table 1. From the ROPT, it was shown that CCGE did not cause any skin reaction. From SCPT, CCGE did neither cause any irritation nor allergic skin reaction at the concentration of $1 \%$ and $3 \%$. However, at the concentration of $5 \%$, it did not cause any irritation in all subjects

Table 1: Result of Safety Evaluation on CCGE in human skin ( $n=50)$

\begin{tabular}{cccccc}
\hline \multirow{2}{*}{ Method(s) } & \multirow{3}{*}{ Concentration } & \multicolumn{4}{c}{ Skin Reaction } \\
\cline { 3 - 6 } & & Irritation & \multicolumn{2}{c}{ Allergic } \\
\cline { 3 - 6 } & $5.0 \%$ & 0 & 0.0 & 0 & 0.0 \\
\hline \multirow{2}{*}{ ROPT } & $1.0 \%$ & 0 & 0.0 & 0 & 0.0 \\
& $3.0 \%$ & 0 & 0.0 & 0 & 0.0 \\
SCPT & $5.0 \%$ & 0 & 0.0 & 2 & 4.0 \\
& & &
\end{tabular}

$\mathrm{N}$ : Number of subject with positive response

Table 3: Influence of CCGE on skin lightening compared to baseline $(\mathrm{n}=\mathbf{2 8})$

\begin{tabular}{cccc}
\multirow{2}{*}{ Product } & \multicolumn{3}{c}{ Skin Melanin Index (unit) } \\
\cline { 2 - 4 } & Baseline & After 2 weeks & After 4 weeks \\
\hline CCGE & $524.19 \pm 3.5$ & $520.62 \pm 3.3 \mathrm{~ns}$ & $521.29 \pm 3.4 \mathrm{~ns}$ \\
Base & $532.9 \pm 3.7$ & $529.8 \pm 3.7$ & $529.9 \pm 3.4$ \\
\hline
\end{tabular}

ns: no significant difference to base was observed ( $>0.05)$ and only caused allergic in $4.0 \%$ of the subjects. As from subjective assessment, none of the subject experiences any uncomfortable sensation.

\section{HETCAM test}

The HETCAM test revealed that at the concentration of 5\% CCGE in demineralised water did not cause any irritation at the ocular level (Table 2).

\section{Evaluation on Skin Melanin Index}

The evaluation of Mexameter showed that CCGE significantly reduce skin melanin index by 3.6 units and 2.9 units after two weeks and four weeks of application respectively. However, these values were not significant to its base (Table 3).

\section{Evaluation on Skin Elasticity and Firmness}

The evaluation using Cutometer showed that application of CCGE increase skin firmness significantly after two weeks and four weeks by 6.2 percent and 18.1 percent (Table 4 ). These values were also significantly higher than its base. The application of CCGE also increase skin elasticity significantly by 5.6 percent and 10.3 percent respectively, which were also significantly higher than its base (Table 5).

Table 2: Result of HETCAM Test on CCGE

\begin{tabular}{ccc} 
Sample & $\begin{array}{c}\text { Mean Irritation Index } \\
(\mathbf{N})^{*}\end{array}$ & Haemorrhage \\
Negative control: $0.9 \% \mathrm{NaCl}$ & 0 & Yes \\
Positive control: $0.5 \%$ & 12 & No \\
$\begin{array}{c}\text { Sodium Lauryl Sulfate } \\
\text { Testing product: CCGE in }\end{array}$ & 0 & \\
demineralized water $(5 \%)$ & & \\
\hline
\end{tabular}

${ }^{*}: \mathrm{N}<1$ (practically non irritant); $1<\mathrm{N}<5$ (slightly irritant); $5<\mathrm{N}<9$ (moderately irritant); $\mathrm{N}>9$ (strongly irritant)

Table 4: Influence of CCGE on skin firmness compared to baseline $(\mathbf{n}=\mathbf{2 8})$

\begin{tabular}{cccc}
\hline \multirow{2}{*}{ Product } & \multicolumn{3}{c}{ Skin Firmness (unit) } \\
\cline { 2 - 4 } & Baseline & After 2 weeks & After 4 weeks \\
\hline CCGE & $0.3645 \pm 0.011$ & $0.3419 \pm 0.014^{\star}$ & $0.2987 \pm 0.012^{\star}$ \\
Base & $0.1858 \pm 0.010$ & $0.1842 \pm 0.089$ & $0.2088 \pm 0.013$ \\
\hline
\end{tabular}

*: significantly winning/losing from baseline at $\mathrm{p}<0.05$

Table 5: Influence of CCGE on skin elasticity compared to baseline $(n=28)$

\begin{tabular}{cccc}
\hline \multirow{2}{*}{ Product } & \multicolumn{3}{c}{ Skin Elasticity (unit) } \\
\cline { 2 - 4 } & Baseline & After 2 weeks & After 4 weeks \\
\hline CCGE & $0.7137 \pm 0.018$ & $0.7535 \pm 0.015^{*}$ & $0.7874 \pm 0.012^{*}$ \\
Base & $0.8674 \pm 0.014$ & $0.8653 \pm 0.013$ & $0.8924 \pm 0.013$ \\
\hline
\end{tabular}

*: significantly winning/losing from baseline at $\mathrm{p}<0.05$ 


\section{CONCLUSION}

Combination of Centella asiatica, Camellia sinensis, and Glycine max extract (CCGE) is proven to be safe on skin application as well as at ocular level. Centella asiatica, Camellia sinensis, and Glycine max extract (CCGE) as combination extract can significantly improve skin firmness and elasticity which are related to aging.

\section{ACKNOWLEDGEMENT}

The Authors are very grateful to PT. Martina Berto, Tbk, Martha Tilaar Group for the financial support provided for this project.

\section{CONFLICT OF INTEREST}

The Authors declare no conflict of interest.

\section{ABBREVIATION USED}

CCGE: Centella asiatica, Carmelia sinensis and Glycerine max Extract; HETCAM: Hen's Egg Test Chorioallantoic Membrane; DPPH: 1,1-Diphenyl-2-picylhadrazyll; ROPT: Repeated Opened Patch Test; SCPT: Single Closed Patch Test.

\section{REFERENCES}

1. Euromonitor Research, Natural ingredients drive growth in cosmetics and toiletries. Available at http://blog.euromonitor.com/2005/09/natural-ingredients-drivegrowth-in-cosmetics-and-toiletries.html

2. Joshi LS, Pawar HA. Herbal Cosmetics and Cosmeceuticals: An Overview. Natural Products Chemistry and Research. 2015;3(2):1-8.

3. Clevastar Study to Learn, 10 Largest Archipelago in the World. Available at https://clevaster.wordpress.com/2015/07/12/10-largest-archipelago-in-theworld/

4. Shimada K, Fujikawa K, Yahara K, Nakamura T. Antioxidative properties of xanthan on the autoxidation of soybean oil in cyclodextrin emulsion. Journal of Agriculture Food Chemistry. 1992;40(6):945-8.

5. Vanni A, Gastaldi D, Giunata G. Kinetic investigation on the double enzyme activity of the tyrosinase mushroom. Annalli di Chimica. 1990;80:35-60.

6. Curry AS, Getting SD, McEwen GN. CTFA safety testing guidelines. The cosmetic, toiletry, and fragrance association. Washington. 1991.

7. SCCNFP. Notes of guidance for testing of cosmetic ingredients for their safety Evaluation. Available at http://ec.europa.eu/health/ph_risk/committees/sccp/ documents/out130_en.pdf

8. Departemen Kesehatan Republik Indonesia. Formularium Kosmetika Indonesia Jakarta: Departemen Kesehatan Republik Indonesia; 1985.

9. Luepke NP. Hen's egg chorioallantoic membrane test for irritation potential. Food Chemistry Toxicity. 1985;23(2):287-91.

10. Maily et al. Efficacy testing of anti aging complex 01 on healthy skin. Jakarta: Corporate Research PT. Martina Berto; 2016. Number of subject with positive responseTable 2: Result of HETCAM Test on CCGEns: no significant difference to base was observed ( $p>0.05)$

Article History: Submission Date: 17-01-17; Revision Date: 13-02-17; Accepted Date: 24-02-17.

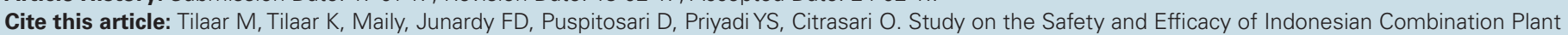
Extract in Cosmetics. J Young Pharm. 2017;9(1)Suppl:s52-s5. 